Available online at GSC Online Press Directory

GSC Biological and Pharmaceutical Sciences

e-ISSN: 2581-3250, CODEN (USA): GBPSC2

(RESEARCH ARTICLE)

\title{
Investigation of the relation between feline infectious peritonitis and retroviruses in cats
}

\author{
Aydin Hakan ${ }^{1, *}$ and Yildirim Serkan ${ }^{2}$ \\ ${ }^{1}$ Faculty of Veterinary Medicine, Department of Virology, 25240, Erzurum, Turkey. \\ 2 Faculty of Veterinary Medicine, Department of Pathology, 25240, Erzurum, Turkey.
}

Publication history: Received on 17 January 2019; revised on 11 February 2019; accepted on 13 February 2019

Article DOI: https://doi.org/10.30574/gscbps.2019.6.2.0011

\begin{abstract}
Feline infectious peritonitis (FIP) infection is a highly pathogenic and fatal systemic inflammatory disease in cats. Although FIP infection is associated with the immune system, there are several predisposing factors of the disease. FIP has a high rate of interaction with other infectious agents. The feline immunodeficiency virus (FIV) and Feline Leukemia Virus (FeLV) infections play a predisposing role by reducing immunity against FIP. This study was conducted with the Turkish Van cats, which is a cat breed specific to the Lake Van region of Turkey, between 2014 and 2015 . Ascites and organ damage symptoms were identified by pathological analysis in 15 Van cats that were brought to the Van Yüzüncü Yll University. Polymerase chain reaction (PCR) was used to investigate the presence of FIV and FeLV in the homogenate of brain, lung, liver, kidney, spleen and intestines tissue samples with lesions. As a result of the analysis, FIV was identified in $1(6.7 \%)$ out of 15 cats, and all cat samples were found to be positive in terms of FeLV (100\%). In the phylogenetic analysis of positive samples, all FeLVs were observed to be in endogenous-FeLVs. In the FIV phylogenetic analysis, our study strain was found to be in the FIV sub-group B. The fact that FIP positive cats were also FeLV positive at the same time has attracted the attention to the pathogenesis of Endogenous-FeLV in malignant diseases, which has not yet been understood. Particularly, it was concluded that the prevalence of FIP infection can be reduced by paying attention to FIV and FeLV vaccination in multiple-cat sheltering environments in young cats.
\end{abstract}

Keywords: FeLV; FIP; FIV; Phylogenetic analysis

\section{Introduction}

Feline infectious peritonitis (FIP) infection is a fatal disease caused by Feline infectious peritonitis virus (FIPV) resulting from mutation of coronavirus (FCoV). While FCoV has an enteric form, FIPV is a pathogenic type with systematic infection. Especially the dramatic increase in the cat population after the World War II and, consequently, housing cats in shelters paves the way to the most important scenario for the emergence of FIP. It is believed that FCoV is propagated in the cat population through this mass housing, resulting in mutated FIPV [1,2]. While it is not common in older cats, FIP infection causes death in young cats of 3-24 months of age, and it is usually the most lethal disease in young cats. There are two types of FIP infection: effusive and granulomatous. While the effusive form is observed mostly, it is the form of inflammatory exudate in the body cavities and granulomatous form affecting the organs is observed [1,3]. As reported in a hypothesis related to the formation of FIP, it is known that viral replication in cats with FCoV occurs in enterocytes. However, another FIP variant has also been reported, which can replicate in macrophages as a result of the mutation in the genome of FCoV [4]. FIP infection is a slow, progressive disease and can last for weeks or months. However, retroviral opportunistic infections such as feline immunodeficiency virus (FIV) and feline leukemia virus (FeLV), which cause immune suppression, make death inevitable by reducing the immunity against FIP and accelerating

\footnotetext{
${ }^{*}$ Corresponding author

E-mail address: hakanaydin.dr@gmail.com
} 
the disease. Even if cats overcome the FIP infection, FIPV reactivation occurs in the case of secondary FeLV and FIV infection, and results in death in a short time [4-6].

The Retroviridae family, which contains immune-suppressing viruses such as FIV and FeLV, has a linear, singlestranded, non-segmented, positive-polarity RNA genome of 8-13 kb in size. Retroviruses, which are morphologically enveloped and have icosahedral symmetry, contain gag, pol and envelope gene regions $[7,8]$.

The immune system plays an important role in the clinic manifestation of FIP (wet/granulomatous). In granulomatous cases (dry FIP), cellular immunity and T-cell mediated humoral immunity are prominent. Especially in the FeLV infections, FeLV infection affect the clinic of FIP infection due to disruptions in this mechanism of immune system [9]. In addition, in cats carrying FIP infection in the form of latent or restricted infection, re-infection may occur as a result of FeLV carriage. This will again result in death through a clinic FIP [5]. FIV infection is one of the most important coinfection factors in the conversion into FIP in cats with FCoV. It has been reported that FCoV can be highly replicable in immunosuppressed cats as a result of FIV infection and that this may be a predisposing factor for FIP through mutation. In FIV and FCoV infected cats, FIV causes a high degree of replication of FCoV and leads to FIP formation in a short time [1]. These retroviruses that disrupt the immune system are the most important clinical indicators in the mutation of FCoV [1]. In the case of FIP-induced immune suppression, the transmission of opportunistic pathogens such as FIV/FeLV may occur $[10,11]$. Although there are currently commercial vaccines for FIV and FeLV, there is no protective vaccination or effective treatment for FIP in Turkey [12].

This study was planned to determine the interactions of FIPV infection and retroviral (FIV/FeLV) co-infection in Van cats, a cat breed native to Turkey.

\section{Material and methods}

The study population consisted of a total of 15 FIP-suspect cats who were brought to the Van Yüzüncü Yll University between 2014 and 2015 for pathological analysis on cat deaths of the Turkish Van cat breed. For virological analysis, tissue samples from the brain, lung, liver, kidney, spleen and intestines were sent to the virology laboratory in the cold chain. For histopathological examinations, tissue samples from all tissues were taken into $10 \%$ formalin solution. After fixation for 48 hours in the formalin solution, samples were washed in running tap water for 10 hours. After passing through alcohol $\left(70^{\circ}, 80^{\circ}, 90^{\circ}, 96^{\circ}\right.$ and $\left.100^{\circ}\right)$ and xylol series in routine tissue follow-up, they were immersed in the paraffin blocks. Four $\mu \mathrm{m}$ thick sections were taken from each block and preparations were made on the glass slide. Preparations for histopathological examination were stained with Hematoxylin-Eosin (HE) and examined by the light microscopy.

\subsection{Immunohistochemical examination}

All sections taken into the adhesive (poly-L-Lysine) slides for immunoperoxidase examination were passed through xylol and alcohol series for deparaffinization and dehydration. Then, they were washed for 5 minutes in distilled water. After this, they were washed with phosphate buffer solution (PBS, pH 7.2) for 5 minutes, and endogenous peroxidase was inactivated by allowing them to stand in $3 \% \mathrm{H}_{2} \mathrm{O}_{2}$ for 3 minutes. After washing for 5-10 min in PBS, they were allowed to incubate for 5 min with Protein block, which is compatible with all primary and secondary antibodies to prevent nonspecific ground staining. At the end of the incubation, excess of the block solution remaining on the tissue sections were discarded and the drops of primary antibodies (VGEF, Caspase 3, Caspase 8, Caspase 9, PCNA, neuronectin, TRPM 1, TRPM 2, $\alpha$ sinokline, apelin) and PBS in the control group were used without washing. In accordance with the primary antibody, they were allowed to stand one night at $+4{ }^{\circ} \mathrm{C}$. After washing twice for $5 \mathrm{~min}$. with PBS, they were incubated for 10-30 min. at room temperature with biotinylated secondary antibody. Sections washed again with PBS were allowed to stand in streptavidin-peroxidase for 10-30 min., then were washed with PBS in the same manner. After washing, 3-3 'Diaminobenzidine (DAB) chromogen was added to the sections, and allowed to stand 5-10 min. according to chromogen intake. After being immersed in Mayer's hematoxylin for floor staining for 1-2 min., they were washed with running water. Then, they were passed through the alcohol and xylol series and covered with lamellae and examined by the light microscope (Leica DM 1000). The sections were evaluated as none (-), mild (+), moderate $(++)$ and severe $(+++)$ according to their immune positivity.

\subsection{Investigation of retroviral infections in FIP positive cats}

In order to investigate the FIV and FeLV DNA in the FIP positive cats, according to pathological analysis, samples of liver, spleen, kidney and brain tissues with lesions were collected. These tissue samples were combined to prepare the homogenate. Using the supernatant from this tissue homogenate, nucleic acid isolation was performed using the commercial kit (GF-1 Nucleic Acid Extraction Kits, Vivantis, Malaysia). The resulting nucleic acid suspension was used 
as the template in PCR for FIV and FeLV analysis. In order to investigate the FIV DNA, nested-PCR was performed using primer pairs specific to the V3-V6 region of the env gene (Table 1) [13]. For FeLV detection, PCR was performed using primer pairs specific to the env gene region of FeLV [14]. Primer sequences and product sizes are given in Table 1.

Table 1 Oligonucleotide details used in the study

\begin{tabular}{|lllll|}
\hline Primer & Sequence 5'-3' & Polarity & Specificity & size (bp) \\
\hline Env Fw & TAY TGG GCC TGT AAC ACY G & + & FeLV & \multirow{2}{*}{508} \\
Env Rv & CGC TGT TTT AGT CTT TCT CTT A & - & FeLV & \\
VE1S & GAG TAG ATA CWT GGT TRC AAG & + & FIV & \multirow{2}{*}{1211} \\
VE1R & CAT CCT AAT TCT TGC ATA GC & - & FIV & \\
nVE2S & CAA AAT GTG GAT GGT GGA AY & + & FIV & \multirow{2}{*}{859} \\
nVE2R & ACC ATT CCW ATA GCA GTR GC & - & FIV & \\
\hline
\end{tabular}

\subsection{Sequence and phylogenetic analysis}

After the gel electrophoresis, FIV/FeLV positive PCR products were subjected to sequence analyses. The raw data obtained after the sequencing were confirmed with GenBank (NCBI National Center for Biotechnology Information/BLAST). Sequences of reference strains and studied samples were aligned with BioEdit (version 7.0.5) program [15]. Phylogenetic analysis was performed using the MEGA (Version 6.0) program with the neighbor-joining method [16].

\section{Results}

\subsection{Pathological findings}

In the necropsy, gray-white granulomatous lesions in the spleen, liver, kidney, intestine, lung and brain organs and serous atrophy were observed in the fat tissues macroscopically (Figure 1). In the effusive form, it was found that there was a severe icteric in the mucous membranes and an effusion fluid of 1-1.5 $\mathrm{l}$ in the abdominal cavity (Figure 2).

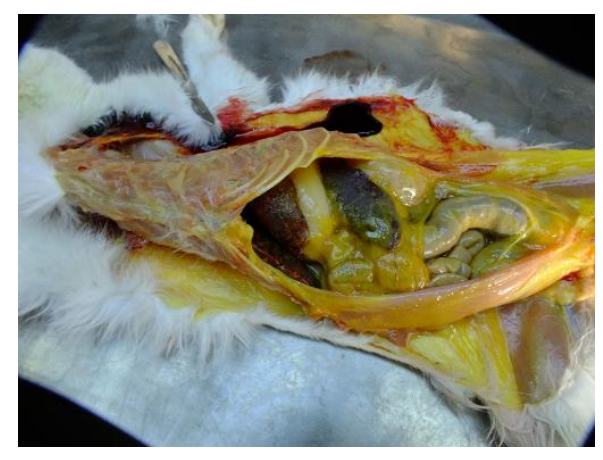

Figure 1 Non-effusive form, pyogranulomas clearly seen in liver, spleen and intestinal serosas

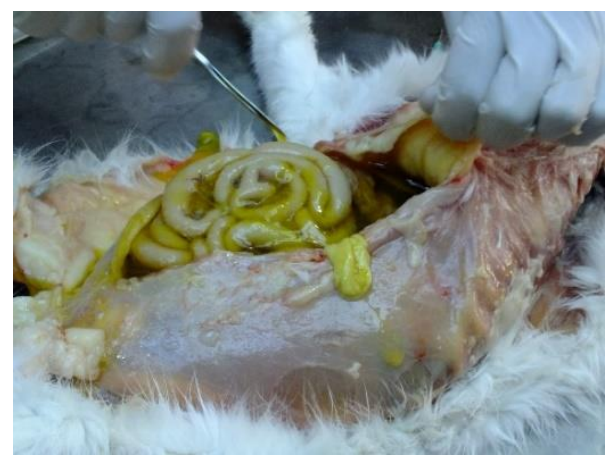

Figure 2 Effusive form, approximately $1.5 \mathrm{l}$ of effusion fluid in abdominal cavity 
The microscopic examination revealed pyogranulomatous lesions, necrotic in the middle, in the spleen, liver, kidney, intestine, lung and brain tissues, especially in the non-effusive form, with neutrophils, macrophages, lymphocytes and plasma cells around (Figure 3). And, vasculitis was identified in the vessels in these organs (Figure 4). While these granules were generally observed near the serosa, it was observed that some of them progressed to the parenchymal tissue especially in the kidneys.

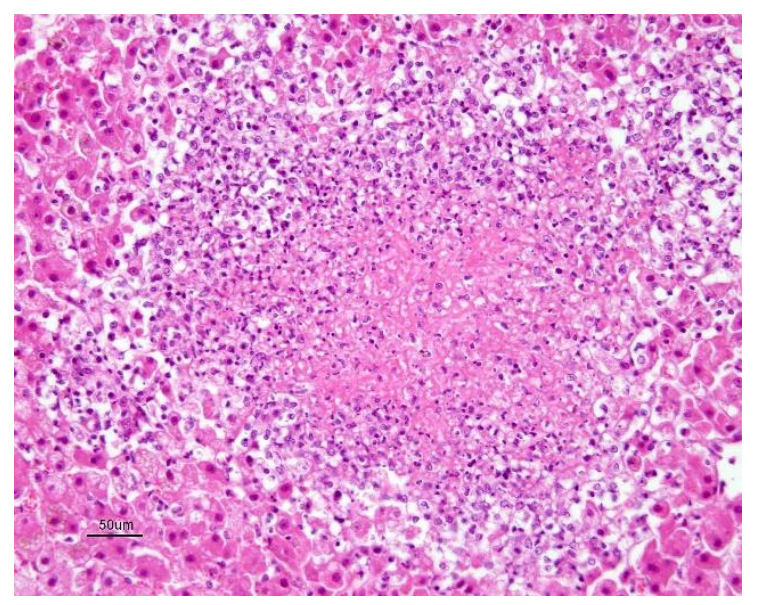

Figure 3 Liver, pyogranuloma, mononuclear cell infiltrations consisting of neurophil leukocytes and mostly with necrotic mass in the middle; H\&E, Bar: $50 \mu \mathrm{m}$

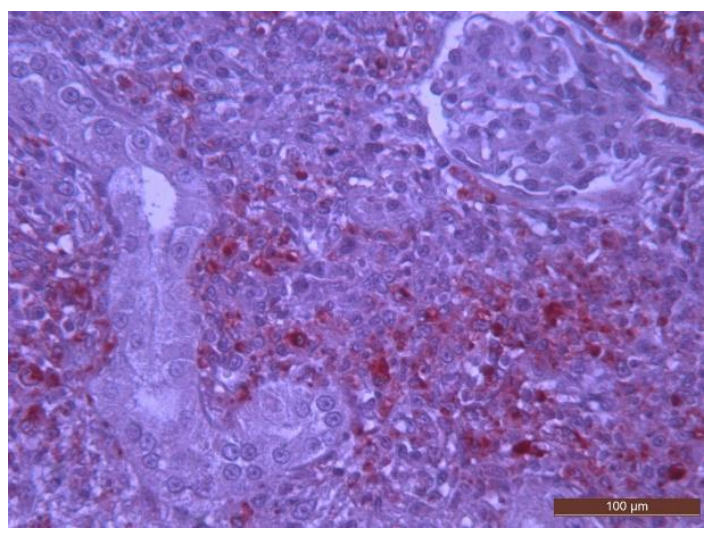

Figure 4 Kidney tissue, FIP positive in tubulus epithelium and interstitial intermittent macrophages, IHC-P, Bar: 100 $\mu \mathrm{m}$

\subsection{Virological findings}

FIV/FeLV DNA was investigated by molecular methods in 15 Van cats diagnosed with FIP by pathological analysis. As a result of PCR analysis, FeLV DNA was detected in all 15 cats (100\%). In the phylogenetic analysis of FeLV strains in our study and reference strains from GenBank, FeLVs clustered two phylogenetic groups: exogenous and endogenous. Sheep and horse retroviruses were used as out-group. In phylogeny, all of our study strains were observed to group together with endogenous-FeLVs (enFeLV) (Figure 5). 


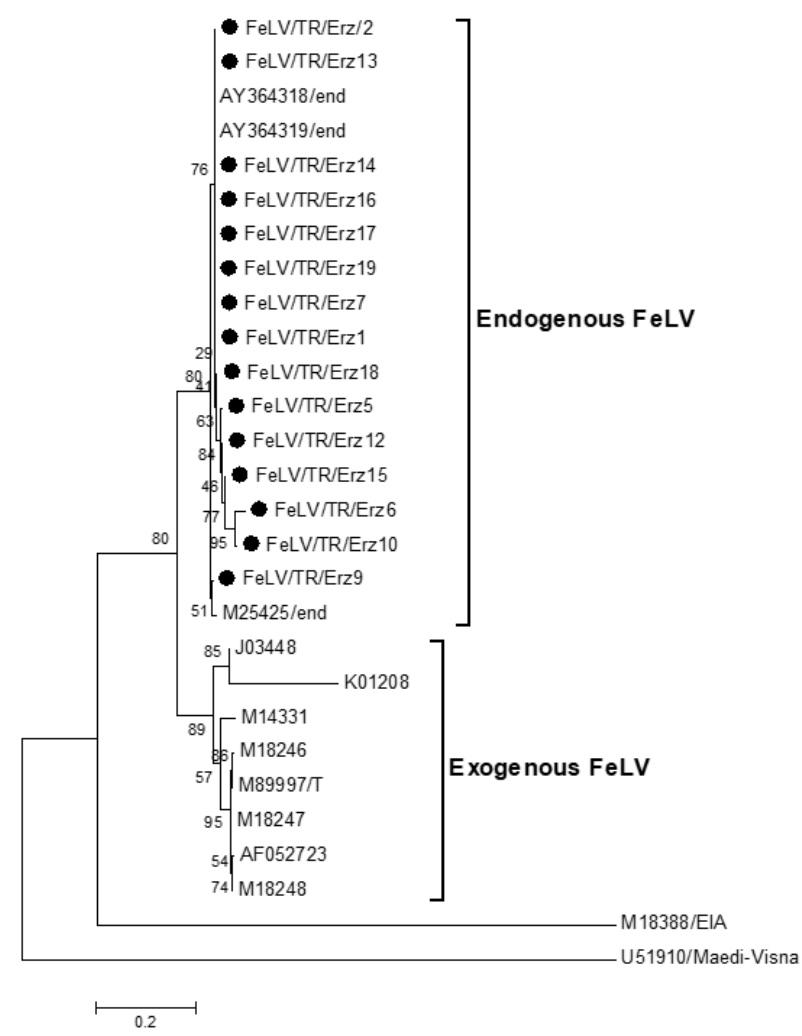

Figure 5 Phylogenetic tree based on a 508 bp nucleotide sequence of 15 strains of FeLV generated by NeighborJoining method, using the MEGA 6.0 software program. Equine and sheep retroviruses were used as an out group. The round symbols $(\bullet)$ indicate Turkish strains

As a result of PCR analysis for FIV, it was identified in 1 (6.7\%) out of 15 samples. Then, sequence reaction and phylogenetic analysis of the FIV strain were performed. The phylogenetic analysis of the FIV strain obtained from our study and the reference strains from GenBank showed that FIV strains were divided into 6 different sub-types ranging from A to F. It was observed that our FIV strain was classified in FIV sub-type B together with Turkish and Japanese strains (Figure 6).

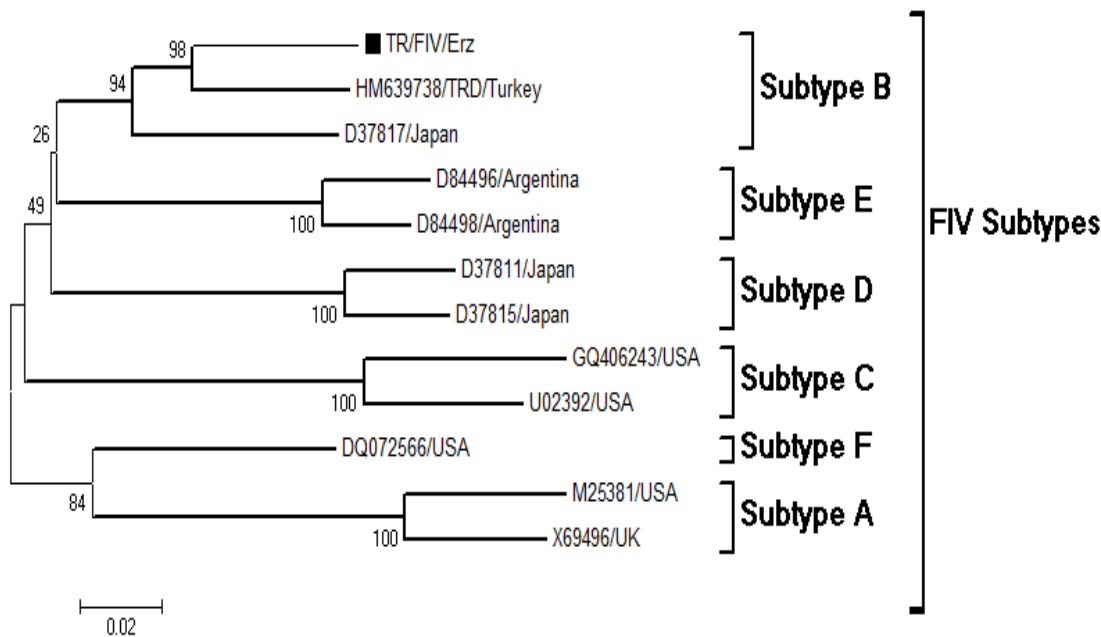

Figure 6 Phylogenetic tree based on a 859 bp nucleotide sequence of FIV strains generated by Neighbor-Joining method, using the MEGA 6.0 software program. The square symbol indicate the Turkish strain 


\section{Discussion}

FIP is a systemic infection associated with the immune system caused by coronaviruses. This infection, which is quite insidious and fatal, is of great importance for domestic and street cats who share the same environment with people and even for cat breeds under protection like Van cats. Eliminating the predisposing factors is the first combat strategy against the FIP infection, which has no effective method of prevention and treatment yet in Turkey. Among these factors, cleaning, hygiene and negative interaction in common housing areas are of importance. In particular, immunosuppressive diseases such as FIV and FeLV are the most important predisposing factors for the emergence of FIPV, a mutational form of coronavirus.

In our study, FIP infection in Van cats, a breed native to Turkey, was revealed by pathological analysis. Then, the presence of FIV and FeLV was investigated in the tissue samples obtained from these cats.

FeLV and FIV are pathogenic infectious agents that cause mortality through immunosuppression in domestic cats. While FIV is similar to human HIV infection, FeLV causes immunosuppressive, neoplastic and hematopoietic disorders in association with subgroups [17]. The retrovirus infections of cats are the most important triggers in the emergence of FIP infection in the areas of cat care houses, shelters and housings. However, a successful result was achieved against retroviral infections, thanks to the aggressive policies of many Western countries, such as test-eradication and vaccination [18]. Although there are vaccines for FIV/FeLV infections in Turkey, an adequate and controlled vaccination program has not been implemented yet.

In our study, FeLV was detected in all cats with FIP. FeLV is divided into 6 groups: A, B, C, D, E and T. These types, called exogenous FeLV, are responsible for leukemia and tumoral formation in cats. Endogenous FeLV (enFeLV) are FeLV sequences transfected into the genome of domestic cats. enFeLV proviruses are found in a variety of organs and tissues, and they perform translation and transcription. However, due to the lack of essential parts of the viral genome, it cannot become an infectious viral particle. However, the infection of cat with exogenous FeLV (field strain) results in the recombination between the two (endogenous/exogenous) and transforms enFeLV into the infectious virus particle. However, it is still unclear whether enFeLV may be responsible for malignant formations in cats with lymphoma, where exogenous strains are not detected [19].

In our study, FeLV was detected in all the tissue suspensions with pathological lesions taken from Van cats. As a result of phylogenetic analysis, it was revealed that all of them were enFeLV. Although this suggests that enFeLV may mutate and cause malignant formations, there is no information to support this in the literature. There are several studies on coronavirus infection of cats in Turkey. In a study conducted by Pratelli et al., FCoV seropositivity was determined in $62 \%$ of cats living in environments such as shelters [20]. In a study conducted by Sahna et al. with molecular methods, the rate of FCoV identified was 54\% [21]. In a study conducted in Turkey, a rare ocular form of FIP infection was identified in cats [22]. In a retrospective study investigating FCoV, FIV and FeLV in 169 sick cats in Turkey, seroprevalence ratios have been determined to be $37 \%, 11 \%$, and $1 \%$ respectively [23]. Similarly, in a study in which FCoV, FIV and FeLV were investigated in chronic gastrointestinal, urinary, respiratory system symptomatic and healthy cats in Turkey, viral agents have been detected by 45.5\%, 9.5\% and 20.5\%, respectively [24]. In our study, FIV and FeLV co-infection were detected in one cat. In the phylogenetic analysis of FIV strain identified in our study, it was found to be of the sub-type B group. In an earlier study conducted with cats in Turkey, sub-type B have been identified in parallel with our results [25]. In a study investigating the FIV and FeLV infections in cats, the rate of FIV and FeLV have been found to be $22.3 \%$ and $5.8 \%$ respectively [26]. Because our study was performed in a specific group, relatively low rates of FIV/FELV were obtained.

\section{Conclusion}

Eliminating the predisposing factors is the first combat strategy against the FIP infection, which has no effective method of prevention and treatment yet in Turkey. Although there are vaccines for FIV/FeLV infections, which are predisposing factors for FIP, an adequate and controlled vaccination program has not been implemented yet. Considering the increased incidence of FCoV and the predisposing factors, vaccination and awareness of cat owners in this regard are important in terms of minimizing the spread of FIPV. It is necessary to determine the countrywide eradication and control policies for agents such as FIPV and FeLV, which pave the way for FIPV infection in cats in shelters and even in cat breeds under protection. 


\section{Compliance with ethical standards}

\section{Acknowledgments}

SY: worked at Van Yüzüncü Yıl University for a while. Cat samples were obtained at that time. SY: provided cat samples and performed pathological analyzes, HA: carried out virological analysis and writing. SY and HA read the entire article and approved.

\section{Disclosure of conflict of interest}

The authors have declared that no conflict of interest.

\section{Statement of ethical approval}

This study were approved by Veterinary Faculty Unit Ethics Committee of Van Yüzüncü Yll University (Protocol no: 2015/03).

\section{References}

[1] Pedersen NC. (2009). A review of feline infectious peritonitis virus infection: 1963-2008. Journal of Feline Medicine and Surgery, 11(4), 225-258.

[2] Felten S, Weider K, Doenges S, Gruendl S, Matiasek K, Hermanns W, Mueller E, Matiasek L, Fischer A, Weber K, Hirschberger J, Wess G and Hartmann K. (2017). Detection of feline coronavirus spike gene mutations as a tool to diagnose feline infectious peritonitis. Journal of Feline Medicine and Surgery, 19(4), 321-335.

[3] Riemer F, Kuehner KA, Ritz S, Sauter-Louis C and Hartmann K. (2016). Clinical and laboratory features of cats with feline infectious peritonitis--a retrospective study of 231 confirmed cases (2000-2010). Journal of Feline Medicine and Surgery, 18(4), 348-356.

[4] Hartmann K. (2017). Feline infectious peritonitis-new developments in pathogenesis, diagnosis, and management. The Thai Journal of Veterinary Medicine, 47, 97-100.

[5] Pedersen NC. (1987). Virologic and immunologic aspects of feline infectious peritonitis virus infection. Advances in Experimental Medicine and Biology, 218, 529-550.

[6] Pedersen N, Evermann J, McKeirnan A and Ott R. (1984). Pathogenicity studies of feline coronavirus isolates 791146 and 79-1683. American journal of veterinary research, 45(12), 2580-2855.

[7] Liu D. (2016). Feline Leukemia Virus. Molecular Detection of Animal Viral Pathogens, First edition. GRC Press. chapter22, 197.

[8] Burmeister T. (2001). Oncogenic retroviruses in animals and humans. Reviews in Medical Virology, 11(6), 369380 .

[9] Hardy WD. (1982). Immunopathology induced by the feline leukemia virus. Springer Seminars in Immunopathology, 5(1), 75-106.

[10] Pedersen N. (1976). Feline infectious peritonitis: something old, something new. Feline practice, 6(3), 42-51.

[11] Cotter SM, Gilmore CE and Rollins C. (1973). Multiple cases of feline leukemia and feline infectious peritonitis in a household. Journal of the American Veterinary Medical Association, 162(12), 1054-1058.

[12] Lewis CS, Porter E, Matthews D, Kipar A, Tasker S, Helps CR and Siddell SG. (2015). Genotyping coronaviruses associated with feline infectious peritonitis. Journal of General Virology, 96(6), 1358-1368.

[13] Nishimura Y, Nakamura S, Goto N, Hasegawa T, Pang H, Goto Y, Kato H, Youn HY, Endo Y, Mizuno T, Momoi Y, Ohno K, Watari T, Tsujimoto H and Hasegawa A. (1996). Molecular characterization of feline immunodeficiency virus genome obtained directly from organs of a naturally infected cat with marked neurological symptoms and encephalitis. Archives of virology, 141(10):1933-1948.

[14] Ramirez H, Autran M, Garcia MM, Carmona MA, Rodriguez C and Martinez HA. (2016). Genotyping of feline leukemia virus in Mexican housecats. Archives Virology, 161(4), 1039-1045. 
[15] Hall TA. (1999). BioEdit: a user-friendly biological sequence alignment editor and analysis program for Windows 95/98/NT. Nucleic acids symposium series: [London]: Information Retrieval Ltd., c1979-c2000.

[16] Tamura K, Stecher G, Peterson D, Filipski A and Kumar S. (2013). MEGA6: Molecular Evolutionary Genetics Analysis version 6.0. Molecular Biology and Evoluation, 30(12), 2725-2729.

[17] Miyazawa T, Ikeda Y, Maeda K, Horimoto T, Tohya Y, Mochizuki M, Vu D, Vu GD, Cu DX, Ono K, Takahashi E and Mikami T. (1998). Seroepidemiological survey of feline retrovirus infections in domestic and leopard cats in northern Vietnam in 1997. Journal of veterinary medical science, 60(11), 1273-1275.

[18] Weijer K, UijtdeHaag F and Osterhaus A. (1986). Control of feline leukaemia virus infection by a removal programme. Veterinary Record, 119(22), 555-556.

[19] Krunic M, Ertl R, Hagen B, Sedlazeck FJ, Hofmann-Lehmann R, von Haeseler A and Klein D. (2015). Decreased expression of endogenous feline leukemia virus in cat lymphomas: a case control study. BMC Veterinary Research, 11, 90.

[20] Pratelli A, Yesilbag K, Siniscalchi M, Yalcm E and Yilmaz Z. (2009). Prevalence of feline coronavirus antibodies in cats in Bursa province, Turkey, by an enzyme-linked immunosorbent assay. Journal of Feline Medicine and Surgery, 11(10), 881-884.

[21] Can-Sahna K, Soydal Ataseven V, Pinar D and Oguzoglu TC. (2007). The detection of feline coronaviruses in blood samples from cats by mRNA RT-PCR. Journal of Feline Medicine and Surgery, 9(5), 369-372.

[22] Baydar E, Eroksuz Y, Timurkan MO and Eroksuz H. (2014). Feline infectious peritonitis with distinct ocular involvement in a cat in Turkey. Kafkas Üniversitesi Veteriner Fakültesi Dergisi, 20(6), 961-965.

[23] Tekelioglu BK, Berriatua E, Turan N, Helps CR, Kocak M and Yilmaz H. (2015). A retrospective clinical and epidemiological study on feline coronavirus (FCoV) in cats in Istanbul, Turkey. Preventive Veterinary Medicine, 119(1-2), 41-47.

[24] Oğuzoğlu T, Muz D, Timurkan M, Maral N and Gurcan I. (2013). Prevalences of feline coronavirus (FCoV), feline leukaemia virus (FeLV), feline immunodeficiency virus (FIV) and feline parvovirus (FPV) among domestic cats in Ankara, Turkey. Revue de médecine vétérinaire, 164(11), 511-516.

[25] Oguzoglu TC, Timurkan MO, Muz D, Kudu A, Numanbayraktaroglu B, Sadak S and Burgu I. (2010). First molecular characterization of feline immunodeficiency virus in Turkey. Archives of Virology, 155(11), 1877-1881.

[26] Yilmaz H, Ilgaz A and Harbour DA. (2000). Prevalence of FIV and FeLV infections in cats in Istanbul. Journal of Feline Medicine and Surgery, 2(1), 69-70.

\section{How to cite this article}

Aydin H and Yildirim S. (2019). Investigation of the relation between feline infectious peritonitis and retroviruses in cats. GSC Biological and Pharmaceutical Sciences, 6(2), 71-78. 